\title{
Study on Microchannel Design and Burst Frequency Detection for Centrifugal Microfluidic System
}

\author{
Yaw-Jen Chang, ${ }^{1}$ Shia-Chung Chen, ${ }^{1,2,3}$ and Cheng-Li Hsu ${ }^{1}$ \\ ${ }^{1}$ Department of Mechanical Engineering, Chung Yuan Christian University, Chung-Li 32023, Taiwan \\ ${ }^{2}$ R\&D Center for Membrane Technology, Chung Yuan Christian University, Chung-Li 32023, Taiwan \\ ${ }^{3}$ ReD Center for Mold and Molding Technology, Chung Yuan Christian University, Chung-Li 32023, Taiwan
}

Correspondence should be addressed to Shia-Chung Chen; shiachun@cycu.edu.tw

Received 19 October 2012; Revised 2 January 2013; Accepted 2 January 2013

Academic Editor: Dachamir Hotza

Copyright (C) 2013 Yaw-Jen Chang et al. This is an open access article distributed under the Creative Commons Attribution License, which permits unrestricted use, distribution, and reproduction in any medium, provided the original work is properly cited.

\begin{abstract}
A centrifugal microfluidic system has been developed in this study, enabling the control and measurement of the burst frequency in order to manipulate the liquid. The radial microfluid chips with different microchannel dimensions were designed for simulation analyses and experimental verifications. The microfluidic flow in the microchannel was analyzed using software CFDRC, providing an accurate result compared with that from experiment. The results show that the design of the overflow microchannel can correctly keep the liquid volume with error as low as $5 \%$. For mercurochrome, the burst frequency has an inverse proportion to the channel width, and the simulation results agree with the experimental results. For oil, however, the experimental and simulation results indicate that the relationship between the burst frequency and channel width is not obvious due to oil properties. Since the simulation approach can provide an accurate prediction of flow behavior in the microchannel, the design of radial microfluid chip and the control of burst frequency can be achieved effectively. A practical application to design the centrifugal microfluidic disc for blood typing test was also carried out in this study. The centrifugal microfluidic system can successfully control the spinning speed to achieve the result of adding reagents in a specific sequence.
\end{abstract}

\section{Introduction}

Biochip is an emerging technology that integrates biology, chemistry, optics, and electronics for biochemical analyses. These devices fabricated using (micro-electro-mechanical system) MEMS technique on glass, plastic, or quartz can conduct an analytical process in microscale volumes. Lab-on-achip (LOC) device, through the manipulation of microfluids, is one of the major types of biochips. Usually, LOC device contains several functional components, such as pump, valve, mixer, and filter, for performing sample preparation, test, and detection in a single chip. LOC devices have been successfully applied to numerous biochemical analysis procedures, including polymerase chain reaction (PCR), immunoassay, biochemical assay, and clinical diagnostics [1].

Since the device is miniaturized, the channel size has been reduced within the microscale. Technique for controlling and observing microfluid becomes the hinge of precise fluid manipulation. Two representative methods for microfluid actuation are electrokinetic and pressure-driven approaches, both of which require external actuation mechanisms to drive the microfluid flow.

Recently, the centrifugal force has been found to be an excellent and elegant method to control fluid flowing from the microchamber near the center of centrifugal disc to the peripheral microchamber. In the centrifugal microfluidic platform, a special component called capillary valve is designed to prevent the fluid from flowing out of the microchamber because of the balance of centrifugal force and capillary force which results from the surface tension in the junction where the microchannel meets the valve. The capillary valve is a kind of passive valve without any moving parts. When the centrifugal force is greater than the capillary force by increasing the spinning speed, the fluid bursts through the valve and begins to flow to the next microchamber. This is called the burst frequency [2]. The advantages of capillary valves include easy flow control and reduced production costs [3-8]. 


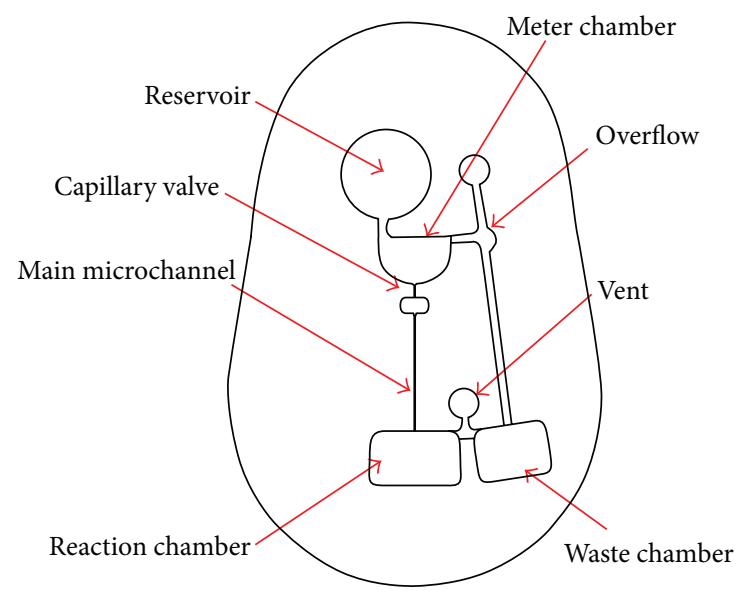

FIGURE 1: Design of radial microfluidic chip.

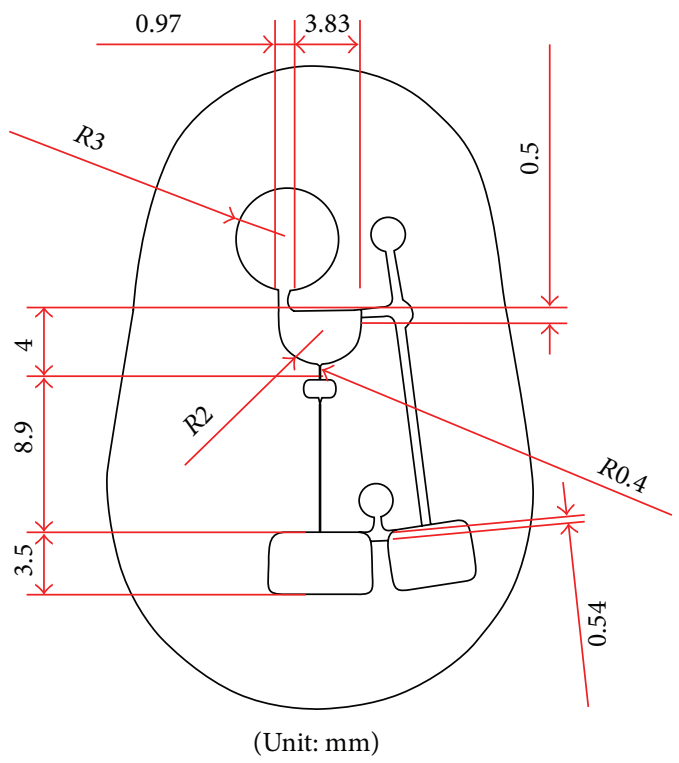

FIGURE 2: Dimensions of radial microfluidic chip.

Numerical simulations have been applied to various fields $[9,10]$, for example, structural mechanics, thermology, electrical science, and hydrodynamics, and so forth. In the design of microfluidic system, simulation is also an economical and widely used solution to study the flow behavior in order to save design time and to reduce design mistakes. The obtained analytic results provide important information such as filling flow front advancement, pressure distribution, and velocity. Thus, this method can offer a simple design guide for the preliminary system design and predict the flow behavior in the microchannel precisely [11-17]. However, the burst frequency of the centrifugal microfluidic system has not been thoroughly investigated. In this study, the simulation approach of burst frequency is discussed, and the results are verified by experiments.

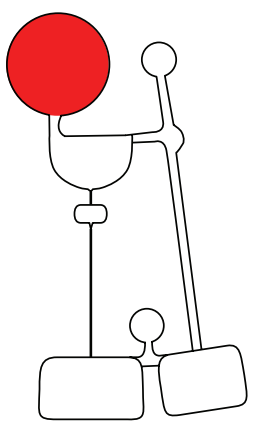

(a)

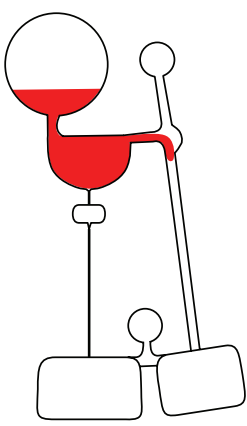

(b)

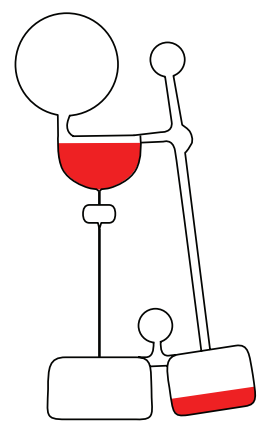

(c)
Figure 3: Design of overflow channel.

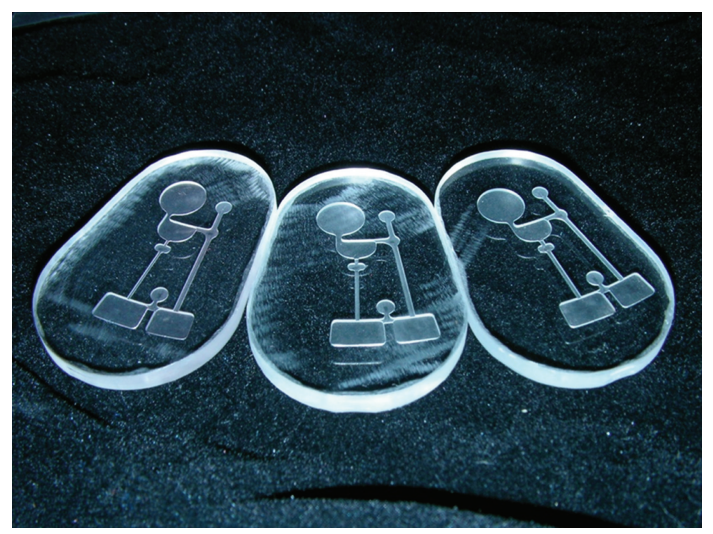

FIGURE 4: Centrifugal microfluidic chips with different widths of main channel.

\section{Chip Design and Simulation}

2.1. Design of Radial Microfluid Chip. The radial microfluid chip designed in this study can be divided into eight elements, including reservoir, meter chamber, capillary valve, main channel, overflow channel, reaction chamber, waste chamber, and vent, as shown in Figure 1. Their dimensions are shown in Figure 2.

(1) Reservoir, Meter Chamber, and Overflow Channel. The fluid is first stored in the reservoir. The meter chamber meters and collects a desired volume of fluid for quantitative experiment. Once the meter chamber is filled with the fluid, the excessive amount of fluid will be drained out from the overflow channel. That is, if the centrifugal force is greater than the surface tension produced in the intersection of overflow channel, the excessive amount of fluid can flow into the waste chamber, as shown in Figure 3. The width of overflow channel is designed to be $500 \mu \mathrm{m}$, and the depth is $200 \mu \mathrm{m}$.

(2) Reaction Chamber and Waste Chamber. The biochemical reaction will take place inside the reaction chamber, while the redundant fluid or the byproducts will be introduced to the waste chamber. The design of these two chambers, including their sizes and locations, relies on the reaction protocol. 
TABLE 1: Basic assumptions and parameters for numerical analyses.

\begin{tabular}{|c|c|}
\hline \multirow{2}{*}{ Problem type } & Flow \\
\hline & Free surface (VOF) \\
\hline \multirow{4}{*}{ Model options } & Transient condition $=$ transient \\
\hline & Rotation reference input \\
\hline & Reference pressure $=100000 \mathrm{~N} / \mathrm{m}^{2}$ \\
\hline & Surface tension \\
\hline \multirow{2}{*}{ Volume conditions } & Secondary fluid \\
\hline & VOF input \\
\hline \multirow{5}{*}{ Boundary conditions } & Inlet $=$ fixed pressure default \\
\hline & Outlet $=$ fixed pressure default \\
\hline & Rotating wall input \\
\hline & Fluid volume fraction \\
\hline & Contact angle for fluid 2 input \\
\hline \multirow{4}{*}{ Initial conditions } & IC applied = volume by volume \\
\hline & Fluid 1 input default \\
\hline & Fluid 2 input default \\
\hline & Volume VOF \\
\hline
\end{tabular}

(3) Capillary Valve. The function of capillary valve is to block the fluid in the microchamber until the spinning speed reaches the burst frequency. Then, the fluid bursts through the valve and begins to flow. Thanks to the design of capillary valves, the different fluids flowing from their respective microchambers can be controlled in a specific sequence. The capillary valve has a sharp increased width in the cross-section of microchannel, producing the resistance of the capillary pressure for the microfluid in capillary valve to stop the flow.

(4) Main Channel. The main channel is used to transport the fluid into the reaction chamber. In this study, different widths of main channel, that is, 300,400 , and $500 \mu \mathrm{m}$, were designed for comparison, as shown in Figure 4.

(5) Vent. The air existing in the sealed microchannel increases the difficulty for fluid actuation. The function of the vent is to exhaust the air in the microchannel so that the fluid can be driven smoothly.

2.2. Modeling and Simulation. Newton's second law states that the time rate of change of the momentum of a fluid element is equal to the sum of the forces on the element. The Navier-Stokes Equation (1) represents the detailed fluid motion through the device as follows:

$$
\begin{gathered}
\frac{\partial(\rho u)}{\partial t}+\nabla \cdot(\rho \vec{V} u)=-\frac{\partial p}{\partial x}+\nabla \cdot(\mu \nabla u)+S_{M x} \\
\frac{\partial(\rho v)}{\partial t}+\nabla \cdot(\rho \vec{V} v)=-\frac{\partial p}{\partial y}+\nabla \cdot(\mu \nabla v)+S_{M y} \\
\frac{\partial(\rho w)}{\partial t}+\nabla \cdot(\rho \vec{V} w)=-\frac{\partial p}{\partial z}+\nabla \cdot(\mu \nabla w)+S_{M z}
\end{gathered}
$$

where $\partial \rho / \partial t$ is the time rate of change of the density, $\nabla \cdot(\rho \vec{V})$ is the net mass flow across the control volume's boundaries and
TABLE 2: The comparison of titrants.

\begin{tabular}{lcccc}
\hline Titrant type & $\begin{array}{c}\text { Density } \\
\left(\mathrm{g} / \mathrm{cm}^{3}\right)\end{array}$ & $\begin{array}{c}\text { Viscosity } \\
\left(\mathrm{mm}^{2} / \mathrm{s}\right)\end{array}$ & $\begin{array}{c}\text { Contact angle } \\
(\mathrm{deg})\end{array}$ & $\begin{array}{c}\text { Surface } \\
\text { tension } \\
(\mathrm{N} / \mathrm{m})\end{array}$ \\
\hline Water & 1.000 & 1.0000 & 65.00 & 0.06800 \\
Mercurochrome & 1.008 & 1.2113 & 70.00 & 0.06763 \\
Oil & 0.850 & 4.2000 & 30.57 & 0.02086 \\
\hline
\end{tabular}

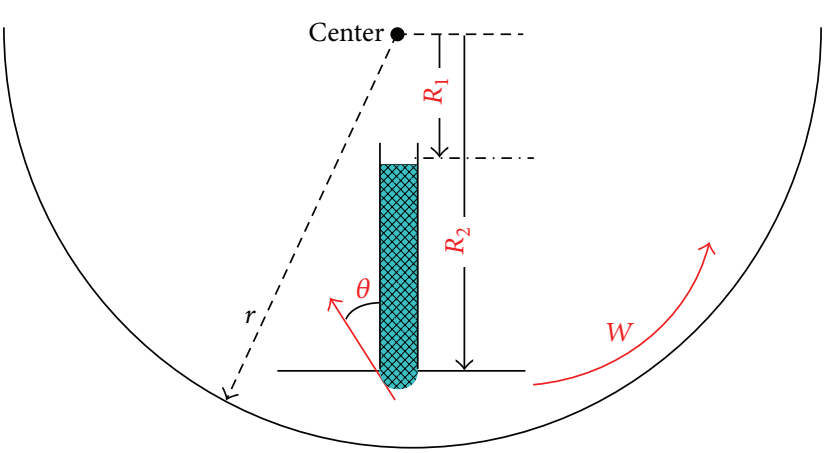

FIgURE 5: Fluid propulsion in centrifugal microfluidics [4].

is called the convective term, $u$ is the velocity of $x$-direction, $p$ is the static pressure, $\mu$ is the kinematic viscosity, and $S_{M x}$ is the body force of $x$-direction. Similar equations can be written for $y$-direction and $z$-direction.

A volume of fluids (VOF) method was used to simulate two different fluid's interface motion in the microchannel. The fluid distribution in the computational grid is accounted for using a single scalar field variable, $F$. This term specifies the fraction of the volume of each computational cell in the grid occupied by fluid two which is water. Thus, $F$ takes the value 1 in cells that contain only fluid two, water, and the value 0 in cells that contain only fluid one which is air. A cell that contains an interface would have a value of $F$ between 0 and 1. The expression is as follows:

$$
\frac{\partial F}{\partial t}+\nabla \cdot \vec{V} F=0
$$

where $F$ is the liquid volume fraction, $t$ is time, $\nabla$ is the standard spatial grad operator, and $\vec{V}$ is the velocity vector.

Under the microscale, the surface tension has significant effect on liquid flow and must be taken into account. Surface tension can be expressed as follows:

$$
\int \Delta p d s=\int \sigma \vec{n} \times d \vec{x} \vec{n}=\nabla F,
$$

where $\Delta p$ is the pressure drop at the free surface, $\sigma$ represents the surface tension, $\nabla F$ is the volume of fluid fraction, $d s$ is an infinitesimal interfacial area, $d \vec{x}$ is the infinitesimal change in the $x$-direction, and $\vec{n}$ is the interface normal.

In this study, computational fluid dynamics (CFD) was employed to simulate microfluidic flow behavior in the microchannel and the burst frequency at the capillary valve. The mesh type, element number, and grid quality are important factors which affect the results and calculation time. 


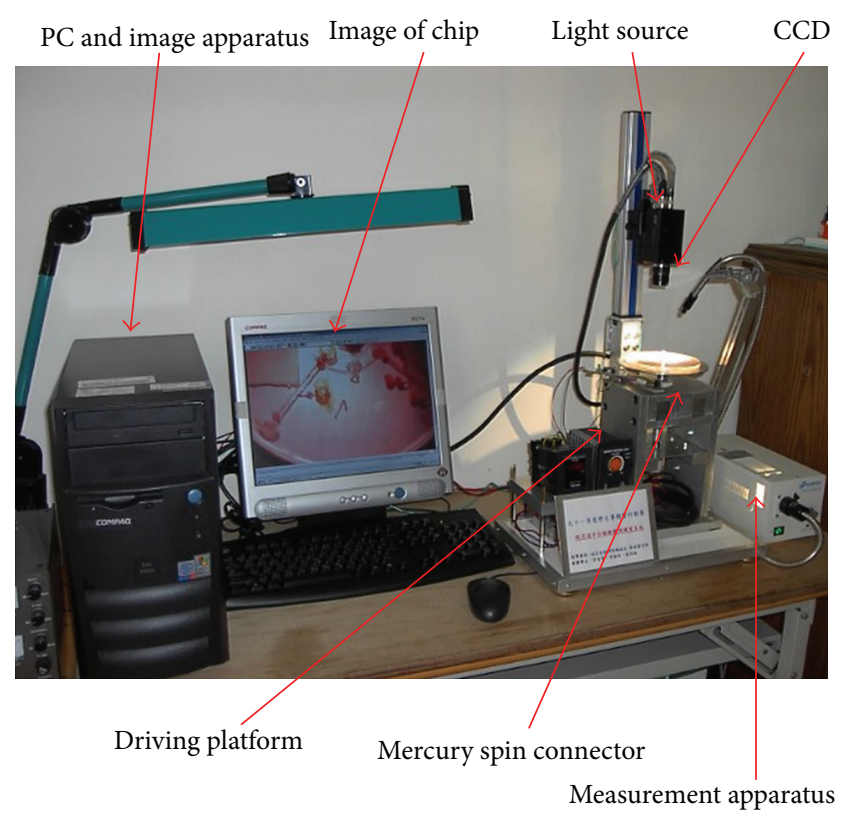

FIGURE 6: Equipment of centrifugal microfluidic system.

Due to the function limitation of software, structured grids are appropriate and used to solve surface tension problems [18]. In order to describe the cross-section of microchannel exactly, a 3D model type was chosen. The microfluid flows into the rectangle channel at a low Reynolds number leading to a flow field which is assumed laminar and uncompressible. Different channel dimensions having widths of 300, 400, and $500 \mu \mathrm{m}$ were studied. Their depths were assumed to be $200 \mu \mathrm{m}$. The distance between the disc center and capillary valve was $50 \mathrm{~mm}$. Other basic assumptions and parameters are listed in Table 1.

The theoretical values of burst frequency can be calculated easily by [4] the following:

$$
f_{b}=\left(\frac{\sigma \cdot \operatorname{Cos} \theta}{\pi^{2} \rho \cdot \Delta R \cdot \bar{R} \cdot D_{H}}\right)^{1 / 2},
$$

where $\Delta R$ is $R_{2}-R_{1}$, and $\bar{R}$ is $\left(R_{2}+R_{1}\right) / 2$ which is best understood through the schematic illustration provided in Figure 5.

2.3. Determination of Fluid Property. Prior to the simulation, the parameters of fluid must be determined. In this study, three types of titrants were chosen as the test fluid to replace the plasma. Since the fluid contacts with the surface of microchannel, the contact angles of the fluid on the substrate material (PMMA) as well as the cover material (adhesive tape) must be measured, respectively. The contact angle, which explains the wetting property of the fluid on the solid surface, was examined using the angle/surface tension meter (KRUSS DSA 10 MK2). Furthermore, viscosity of fluid affecting the flow kinematics is another important parameter. Then, the kinematic viscosity was measured through a viscometer (SVM 3000, Anton Paar).
TABLE 3: The measurements of microchannels.

\begin{tabular}{lccc}
\hline Width & Average width & Depth & Surface roughness \\
\hline 300 & 310 & 170 & 1.5 \\
400 & 400 & 190 & 1.5 \\
500 & 490 & 210 & 2.0 \\
\hline
\end{tabular}

(Unit: $\mu \mathrm{m}$ ).

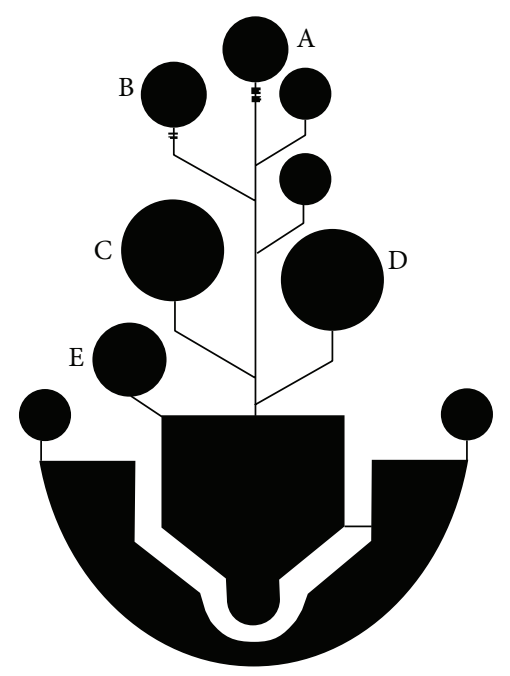

FIGURE 7: Chip design for blood typing test.

Using the measured parameters, the simulation can mimic the real characteristics of the titrants flowing in the microchannel. The properties of these titrants are shown in Table 2. Among these titrants, mercurochrome has properties similar to the plasma.

\section{Fabrication and Experiments}

3.1. Fabrication of Prototype. There are many techniques to manufacture the radial microfluid chip, for example, etching, soft lithography, and computerized numerically controlled (CNC) milling. For the preliminary stage, the CNC milling gains advantages, including low-cost and time-saving advantages, over other techniques. However, it has mechanical limitations, such as the feature size (the channel width is constrained to the diameter of cutter which is greater than $200 \mu \mathrm{m}$ ), dimension control (often 10\% smaller than design), surface quality (the surface roughness is around several microns), and aspect ratio (the ratio of height to width is less than 2). In this study, the material of chip is PMMA.

3.2. Experiment Apparatuses. Experiment apparatuses used in this study include the centrifugal microfluid system, surface tension/contact angle meter, 3D microscope, viscometer, and the pipet. The centrifugal microfluid system is mainly to provide the required centrifugal force to drive the fluid and observe the flow behavior in both microchannels and microchambers. The system comprises a radial microfluid 


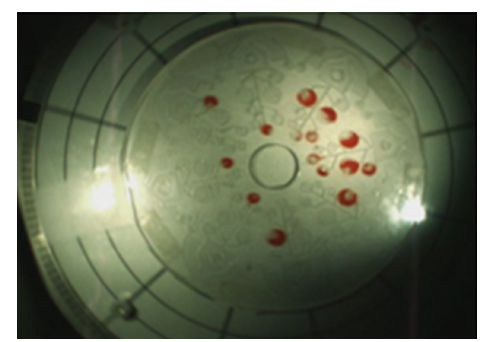

(a) The initial state

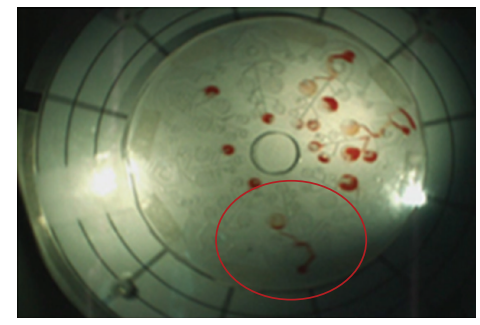

(c) C position, $948 \mathrm{rpm}$

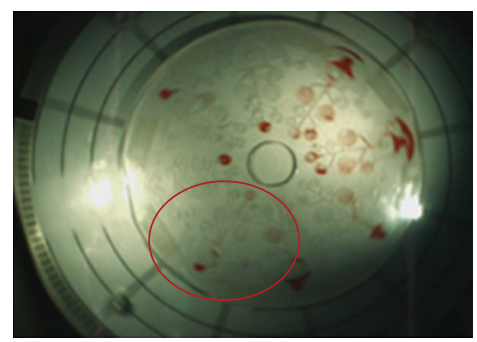

(e) A position, $1176 \mathrm{rpm}$

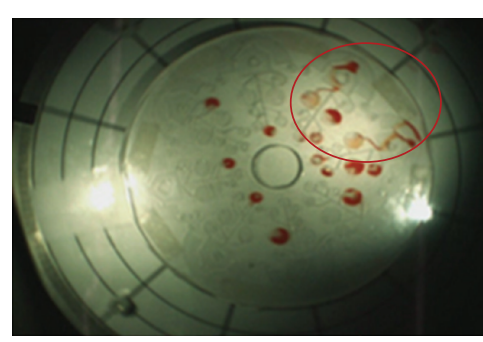

(b) D position, $730 \mathrm{rpm}$

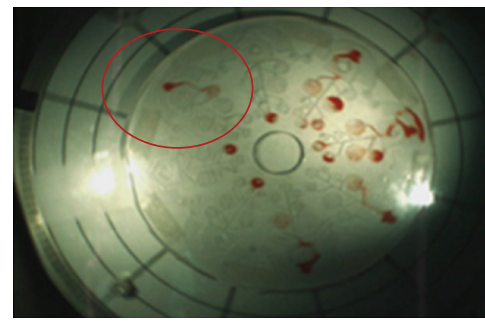

(d) E position, $1070 \mathrm{rpm}$

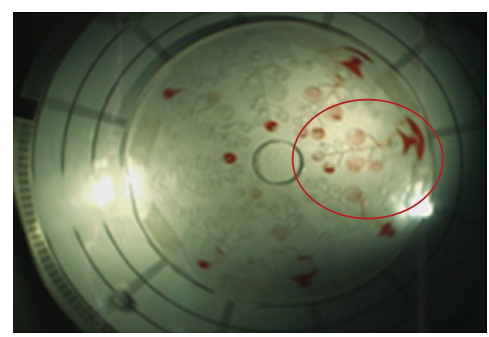

(f) B position, $1312 \mathrm{rpm}$

FIGURE 8: Flowing sequence controlled by the spinning speed.

disc and motor, a CCD camera, and a computer for displaying the images, as shown in Figure 6.

By spinning the radial microfluid chip, the fluid is propelled from the reservoir to the next chamber when the centrifugal force overcomes the surface tension. To observe and measure the burst frequency, the rotating speed of motor was gradually increased by $15 \mathrm{rpm}$ every experimental step. Moreover, at every revolution of the radial microfluid chip a signal is triggered by the sensor and sent to the CCD camera for capturing the image. Therefore, the microfluidic flow behavior can be monitored using the CCD camera and recorded in the computer.

\subsection{Measurement of Chip Dimensions and Titrant Properties.}

The accuracy of microfeatures of this CNC-manufactured radial microfluid chip, including the width and depth of the microchannels, was examined by a 3D microscope with a maximum magnification of 16000 (Keyence Corp., Japan). Five measurements were recorded at each position, and the average values from these five measurements were used to compare with the dimensions of the design model. These measurements are described in Table 3. In addition, the measurements of surface roughness of the bottom surface of the channel are also provided in Table 3.

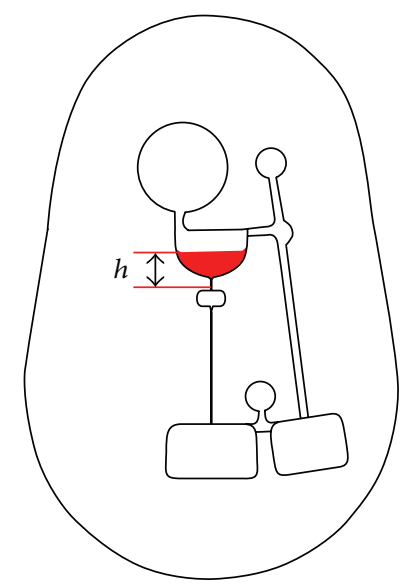

FIgURE 9: The definition of liquid height.

\section{Case Study}

The centrifugal microfluidic system uses the centrifugal force to drive and control the microfluids through a variety of burst frequencies. Hence, the radius centrifugal disc with an array of microchannels offers many significant advantages, such as 


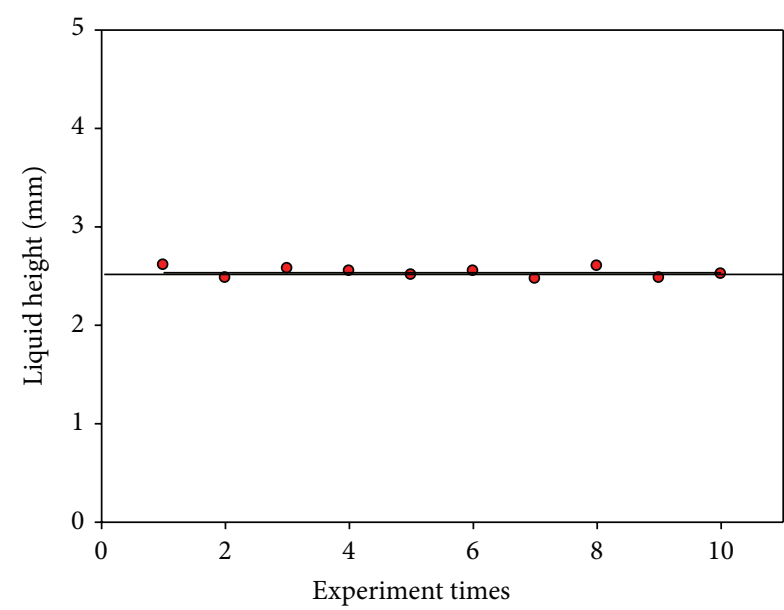

- Liquid height

- Average liquid height

(a) Titrant volume is $1.5 \mathrm{~mL}$

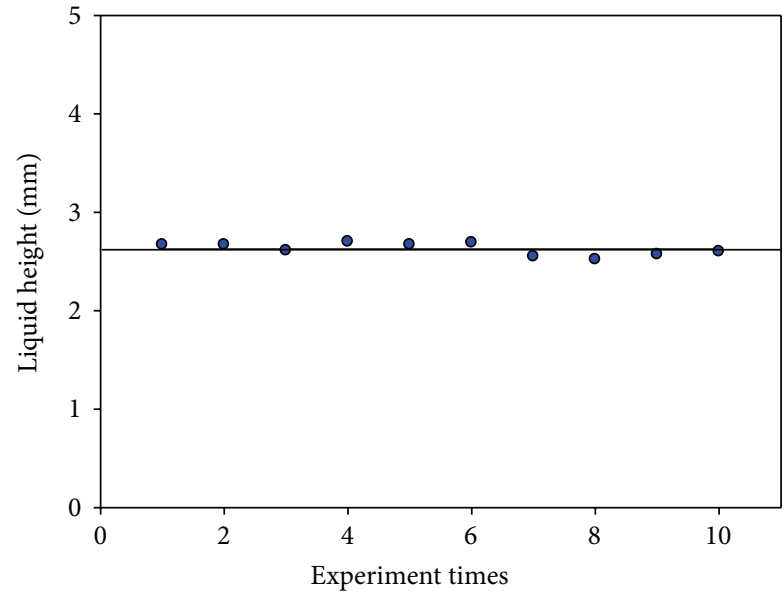

- Liquid height

- Average liquid height

(b) Titrant volume is $2.0 \mathrm{~mL}$

FIGURE 10: Liquid height via different titrant volumes.

small reagent consumption, high throughput due to parallel processing, and short reaction time. Moreover, the automated system reduces tedious and laborious manual operations.

In this study, the centrifugal microfluidic technology was used to design microchannels for blood typing test. Blood typing is a critical test in many medical procedures, especially for blood transfusion. It is based on the antigen-antibody interaction. Because the test protocol of blood typing involves a series of mixing and incubation steps, these reagents must be added in a specific sequence. Figure 7 shows the chip design.

The experimental fluid used in this study was the mercurochrome. We examined whether the fluid injected in the reservoirs (denoted as $\mathrm{A} \sim \mathrm{E}$ in Figure 7) can be driven one by one into the reaction chamber. Obviously, the fluid in reservoir $\mathrm{E}$ must be transported first because it is close to the reaction chamber. That is, the flowing order starts from reservoir $\mathrm{E}$ to reservoir $\mathrm{A}$ and relies on the different burst frequencies. If we cannot obtain distinct burst frequencies, the fluids cannot be controlled in appropriate flowing sequence. That is, those fluids with similar burst frequencies will flow to the reaction chamber simultaneously.

Figure 8 shows a series of experimental pictures. The centrifugal microfluidic system can successfully control the spinning speed to achieve the result of adding reagents in a specific sequence.

\section{Results and Discussions}

(1) Geometry designs of chambers and channels contribute to the control of constant experimental titrant volume. In this study, the purpose of testing the meter chamber and overflow channel is to achieve the quantitative experiments $(1.5 \mathrm{~mL}$ and $2.5 \mathrm{~mL})$. The liquid height is defined as the distance between

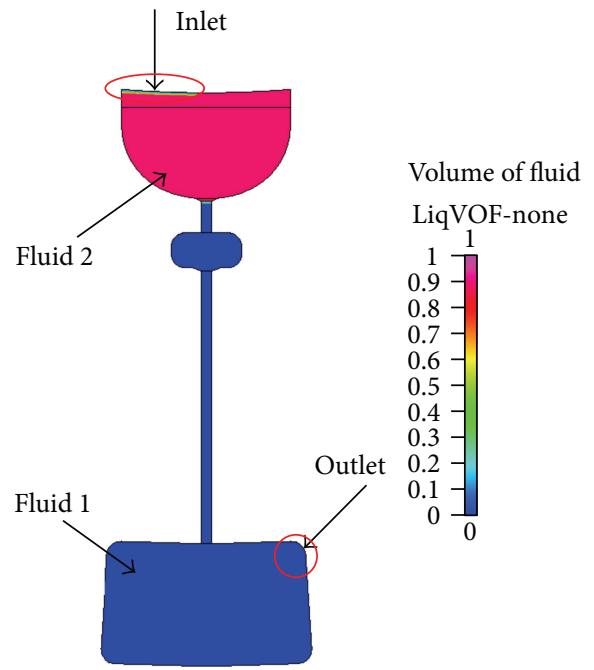

FIGURE 11: Analysis of simplified simulation model.

the lowest liquid position in the meter chamber and the capillary valve, as shown in Figure 9. Through quantitative experiments, the average liquid heights for titrant volumes of $1.5 \mathrm{~mL}$ and $2.5 \mathrm{~mL}$ are $2.54 \mathrm{~mm}$ and $2.62 \mathrm{~mm}$, respectively. However, the design goal of liquid height is $4 \mathrm{~mm}$. The actual liquid height measured from the experiments is about $1.38 \mathrm{~mm}$ less than the target, explaining that the amount of fluid drained out from the overflow channel is more than what we expect. The results might come from the dimension change of meter chamber during fabrication, the viscosity of fluid, and the sealing problem. Nevertheless, for both geometry designs $(1.5 \mathrm{~mL}$ and $2.5 \mathrm{~mL})$, the errors of liquid height deviating from their respective average values in 10 experiments are less than 5\%, 


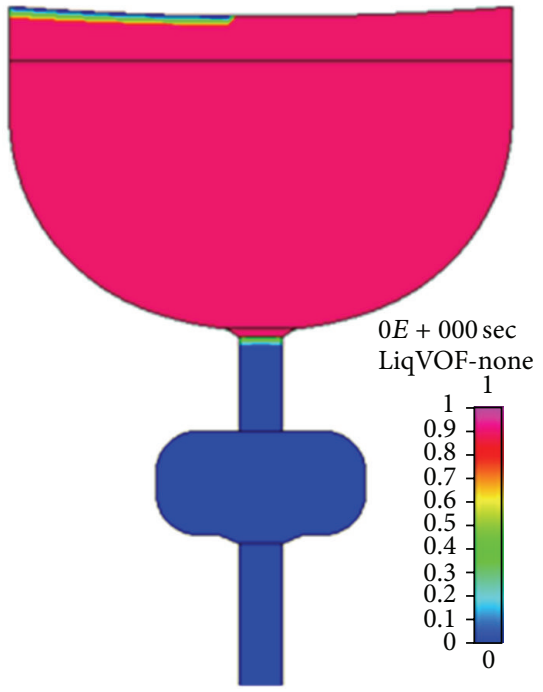

(a) Before the centrifugal force is applied

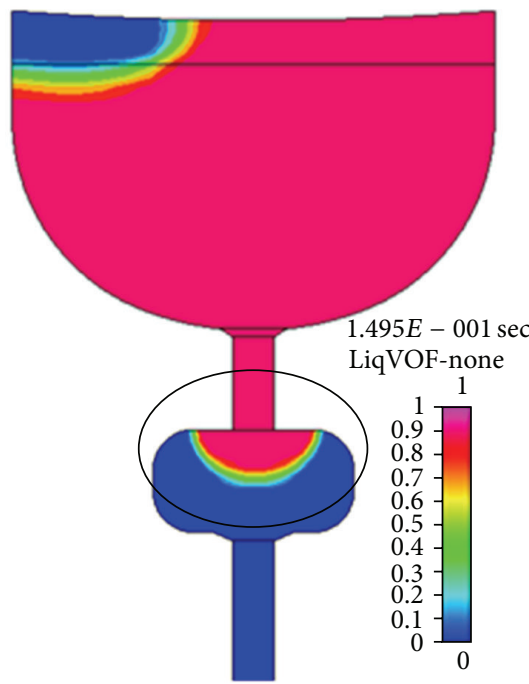

(c) Flow through the switch when burst frequency is overcome

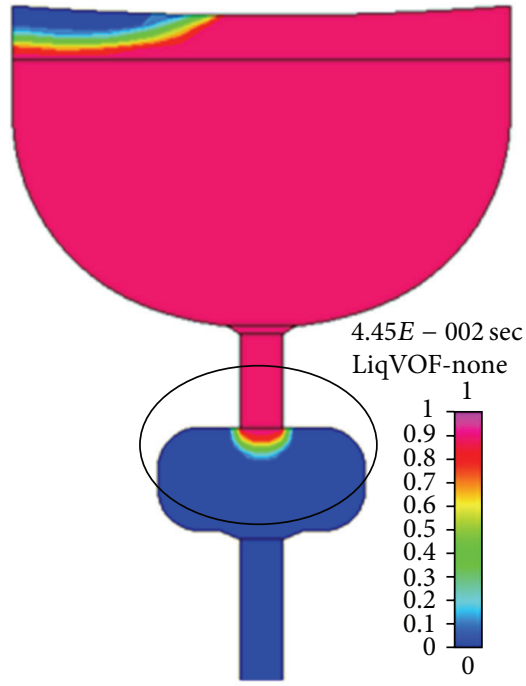

(b) Flow condition near the switch

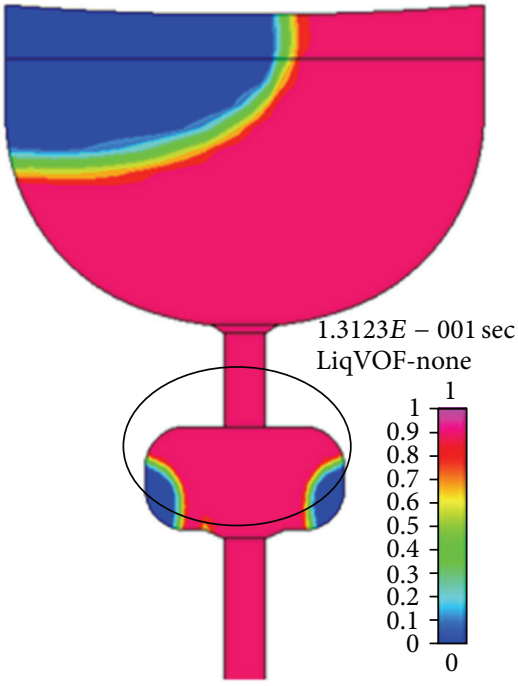

(d) Flow into the major channel

FIGURE 12: Flow condition at different positions simulated by CFDRC.

as shown in Figure 10. This fact eliminates doubt to obtain constant titrant volume with the design.

(2) Compared with the theoretical value, the burst frequency obtained from experiments is $6 \%$ less. For this reason, a shape factor is indeed necessary for burst frequency correction. However, simulation can be applied to biochip design because of the coincidence between the simulation and the experiment. Numerical analyses on centrifugal microfluidic biochip can be established successfully. Moreover, the experimental results agree with the numerical results of the simplified model shown in Figure 11. Using this simplified model, Figure 12 shows the flow before and after the centrifugal force is applied. The burst frequency decreases with a larger hydrodynamic diameter when the titrant is mercurochrome, as shown in Figure 13.
(3) In this study, oil bursting the capillary valve easily even when a driving force is not observed, as shown in Figure 14. A titrant with oil-like properties indicates small contact angle and surface tension. Therefore, the property of oily titrant becomes an important factor for the design of centrifugal microfluidic chip.

(4) Burst frequency decreases with a lower fluid viscosity (direct proportion) and with a deeper chamber (inverse proportion), as shown in Figures 15 and 16 , respectively. The higher viscosity a fluid has, the stronger cohesion forces of molecules exist. Thus, a larger driving force is needed to manipulate this fluid, resulting in higher burst frequency. On the other hand, a deeper chamber allows storing more mass of fluid. Under the same spinning speed, a larger centrifugal force is produced, leading to lower burst 


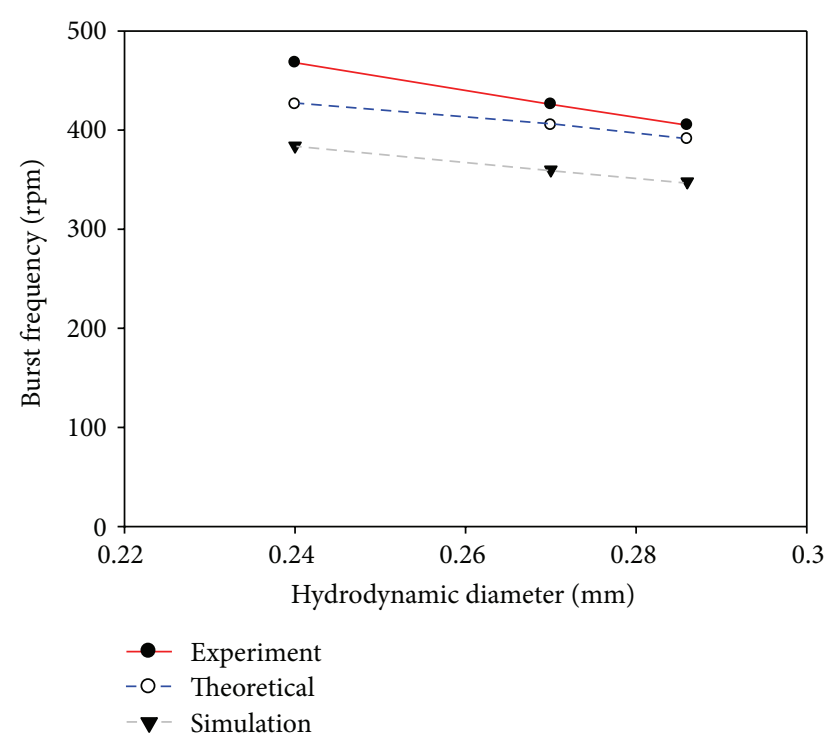

FIGURE 13: Burst frequency decreases with a larger hydrodynamic diameter when the titrant is mercurochrome.

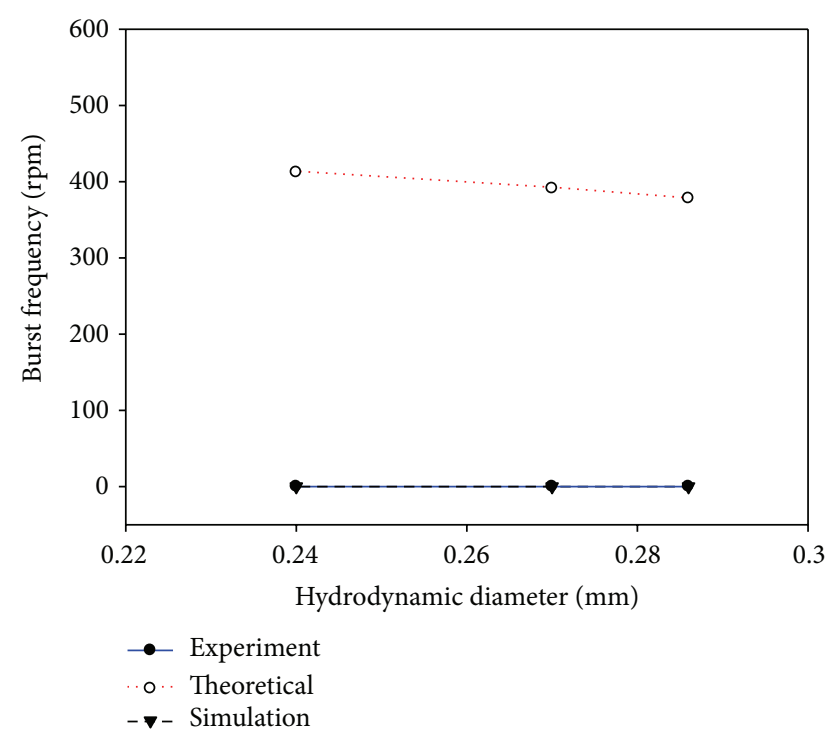

FIGURE 14: Burst frequency varies with a larger hydrodynamic diameter when the type of titrant is oil.

frequency. This experimental result contributes to the design of a correct geometry and to the consideration of the fluid viscosity for obtaining desired burst frequency.

\section{Conclusions}

The computer-aided engineering (CAE) has several advantages, such as quality prediction before manufacturing, problem diagnoses, and problem solving after manufacturing. The CAE has been applied to the development of lab-on-achip technology, which would produce an effective design and enhance the biochip accuracy. In this research, the CAE

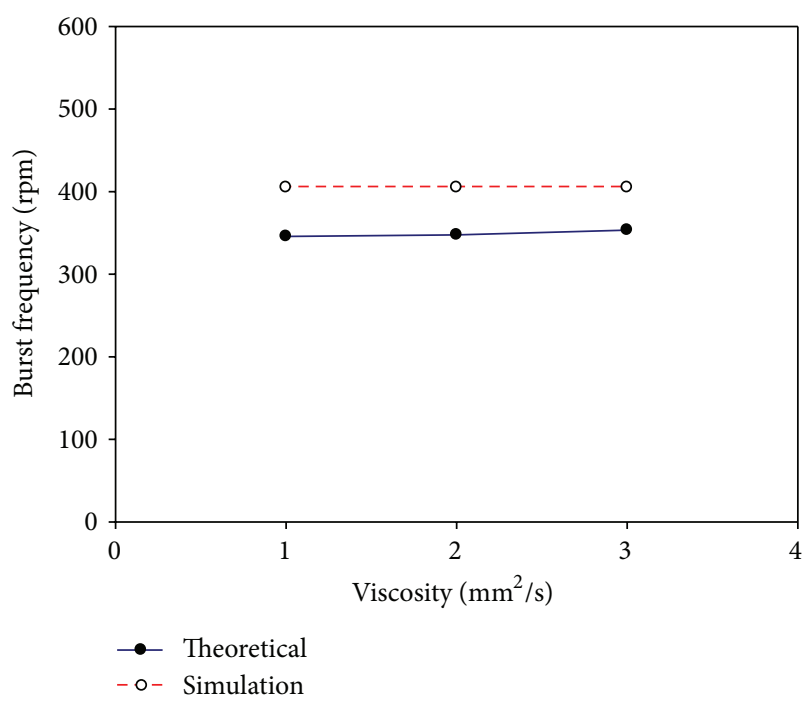

FIGURE 15: Burst frequency decreases with a lower fluid viscosity when the type of titrant is mercurochrome.

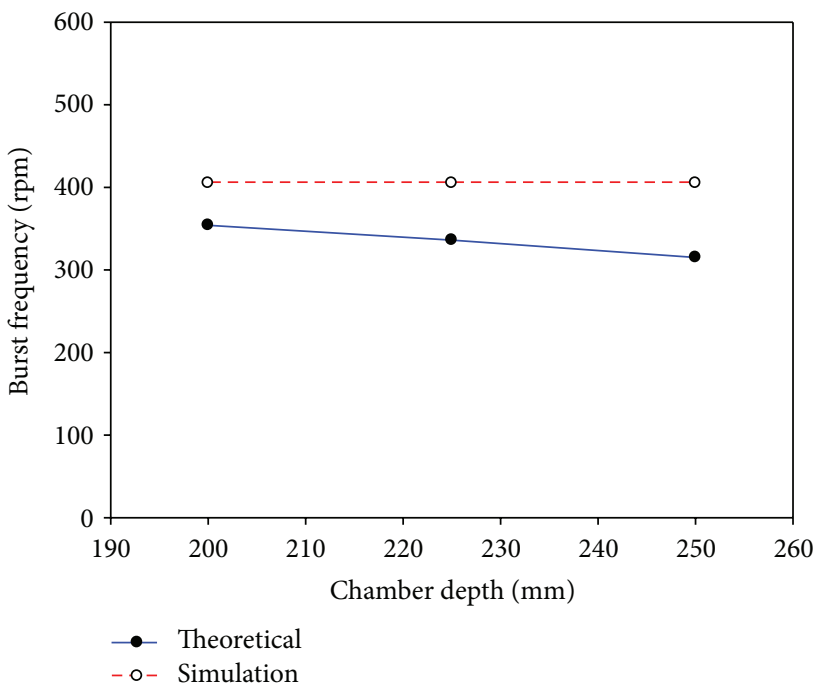

FIGURE 16: Burst frequency decreases with a deeper chamber when the type of titrant is mercurochrome.

software (computational fluid dynamic research corporation, (CFDRC)) was adopted to simulate the burst frequency of capillary valve and liquid flow situation with a centrifugal force (state/system). The standard inspection is established successfully while enhancing the software stability for estimating the microchannel design.

In this study, the burst frequency of a centrifugal force was designed and verified by experimental studies and numerical simulation. From the research results, the following conclusions have been obtained.

(1) The design of overflow chambers and channels on the biochip could control the examined liquid precisely (more stable). From the results of the $1.5 \mathrm{~mL}$ and the $2.0 \mathrm{~mL}$ overflow chamber, the tolerance is less than 
$5 \%$. Even for the different titrated volume, the design could achieve a stable reactive liquid.

(2) Since there is less than 6\% tolerance in comparison between corrected results of burst frequency theory and the one of experimental results, a shape factor can be used for the burst frequency of microfluid by centrifugal force. This work has shown that the experimental and theoretical trends agree, leading to a well-established simulation of the centrifugal microchannel biochip which can be efficiently applied through the design and organization of the biochip.

(3) Due to the specific property of working oil, it is not necessary for a capillary switch design. The research results indicate that the selection of a liquid, resembling the working oil, with a low contact angle and a low surface tension will be in need of consideration due to their dependence on the reliability of the capillary switch.

(4) This study shows that the decrease of the burst frequency is directly related to the lower viscosity and the deeper balanced chamber. This can be utilized in the correction of burst frequency and the design of the biochip by diverse viscosity and in the depth of the balanced chamber.

(5) Through the case study, the centrifugal force driven microfluidic system and the microchannel design can be applied to improve the existing centrifugal system and the development of automated inspection systems for multichannel CD.

\section{Acknowledgment}

This research was financially supported by the National Science Council (NSC 100-2321-B-033-003-), Taiwan.

\section{References}

[1] M. J. Madou, Fundamentals of Microfabrication, CRC Press, Boca Raton, Fla, USA, 2nd edition, 2002.

[2] P. F. Man, C. H. Mastrangelo, M. A. Burns, and D. T. Burke, "Microfabricated capillarity-driven stop valve and sample injector," in Proceedings of the IEEE 11th Annual International Workshop on Micro Electro Mechanical Systems, pp. 45-50, January 1998.

[3] M. A. Burns, B. N. Johnson, S. N. Brahmasandra et al., "An integrated nanoliter DNA analysis device," Science, vol. 282, no. 5388, pp. 484-487, 1998.

[4] M. J. Madou, L. J. Lee, K. W. Koelling, and S. Daunert, "Design and fabrication of polymer microfluidic platforms for biomedical applications," in Proceedings of the 59th Annual Technical Conference (ANTEC), vol. 3, pp. 2534-2538, Society of Plastics Engineers (SPE), 2001.

[5] M. J. Madou, L. J. Lee, S. Daunert, S. Lai, and C. H. Shih, "Design and fabrication of CD-like microfluidic platforms for diagnostic: mircofluidic functions," Biomedical Microdevices, vol. 3, no. 3, pp. 245-254, 2001.
[6] L. J. Lee, M. J. Madou, K. W. Koelling et al., "Design and fabrication of CD-like microfluidic platforms for diagnostics: polymerbased microfabrication," Biomedical Microdevices, vol. 3, no. 4, pp. 339-351, 2001.

[7] R. D. Johnson, I. H. A. Badr, G. Barrett et al., "Development of a fully integrated analysis system for ions based on ion-selective optodes and centrifugal microfluidics," Analytical Chemistry, vol. 73, no. 16, pp. 3940-3946, 2001.

[8] M. J. Madou, Y. Lu, S. Lai, C. G. Koh, L. J. Lee, and B. R. Wenner, "A novel design on a CD disc for 2-point calibration measurement," Sensors and Actuators A, vol. 91, pp. 301-306, 2001.

[9] D. S. Kim, K. C. Lee, T. H. Kwon, and S. S. Lee, "Transient filling flow into microchannels considering surface tension," in Proceedings of the International Conference on Modeling and Simulation of Microsystems (MSM '02), pp. 108-111, April 2002.

[10] D. S. Kim and T. H. Kwon, "Patterning of flow and mixing in rotating radial microchannels," Microfluidics and Nanofluidics, vol. 2, no. 2, pp. 97-105, 2006.

[11] J. Ducrée, T. Brenner, T. Glatzel, and R. Zengerle, "Ultrafast micromixing by coriolis-induced multi-lamination of centrifugal flow," in Proceedings of the International Conference on New Actuators, pp. 533-536, 2004.

[12] T. Brenner, T. Glatzel, R. Zengerle, and J. Ducrée, "Frequencydependent transversal flow control in centrifugal microfluidics," Lab on a Chip, vol. 5, no. 2, pp. 146-150, 2005.

[13] T. Brenner, Polymer Fabrication and Microfluidic Unit Operations for Medical Diagnostics on a Rotating Disk [Ph.D. thesis], University of Freiburg-IMTEK, Baden-Württemberg, Germany, 2005.

[14] D. C. Duffy, H. L. Gillis, J. Lin Jr., N. F. Sheppard, and G. J. Kellogg, "Microfabricated centrifugal microfluidic systems: characterization and multiple enzymatic assays," Analytical Chemistry, vol. 71, no. 20, pp. 4669-4678, 1999.

[15] J. Zeng, D. Banerjee, M. Deshpande, J. R. Gilbert, D. C. Duffy, and G. J. Kellogg, "Design analyses of capillary burst valves," in Proceedings of the Micro-Total Analyses Systems Conference, $\mathrm{p}$. 579, Amsterdam, The Netherlands, May 2000.

[16] J. Zeng, M. Deshpande, K. B. Greiner, and J. R. Gilbert:, "Fluidic capacitance model of capillary-driven stop valves," in Proceedings of the ASME, Orlando, Fla, USA, 2000.

[17] V. B. Makhijani, A. J. Reich, A. Puntambekar, C. Hong, and C. Ahn, "Simulation of flow in structurally programmable microfluidic channels," in Proceedings of the International Conference on Modeling and Simulation of Microsystems, pp. 266269, March 2001.

[18] CFD User Manual, 2003. 

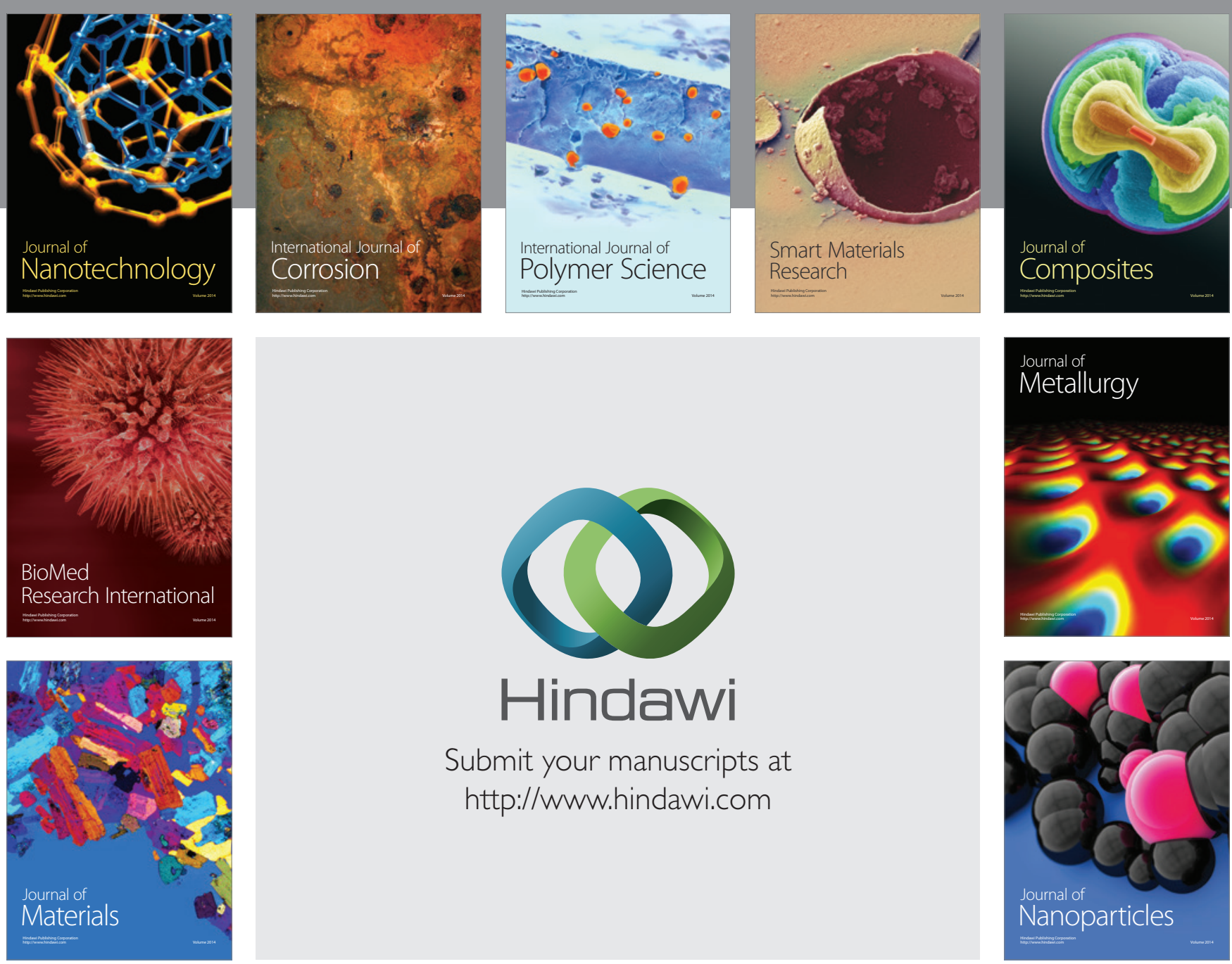

Submit your manuscripts at http://www.hindawi.com
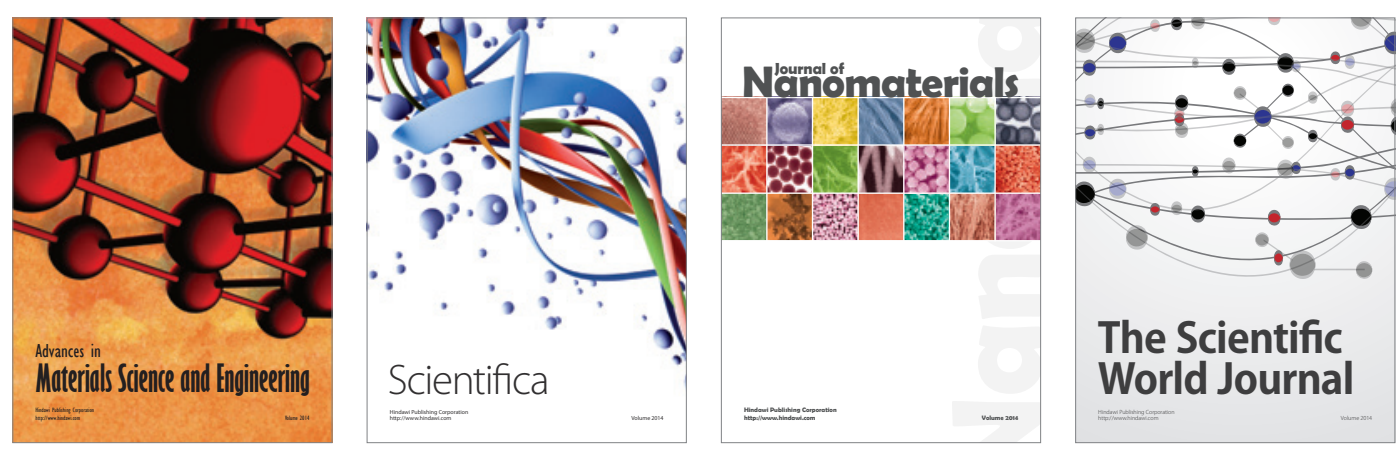

\section{The Scientific World Journal}
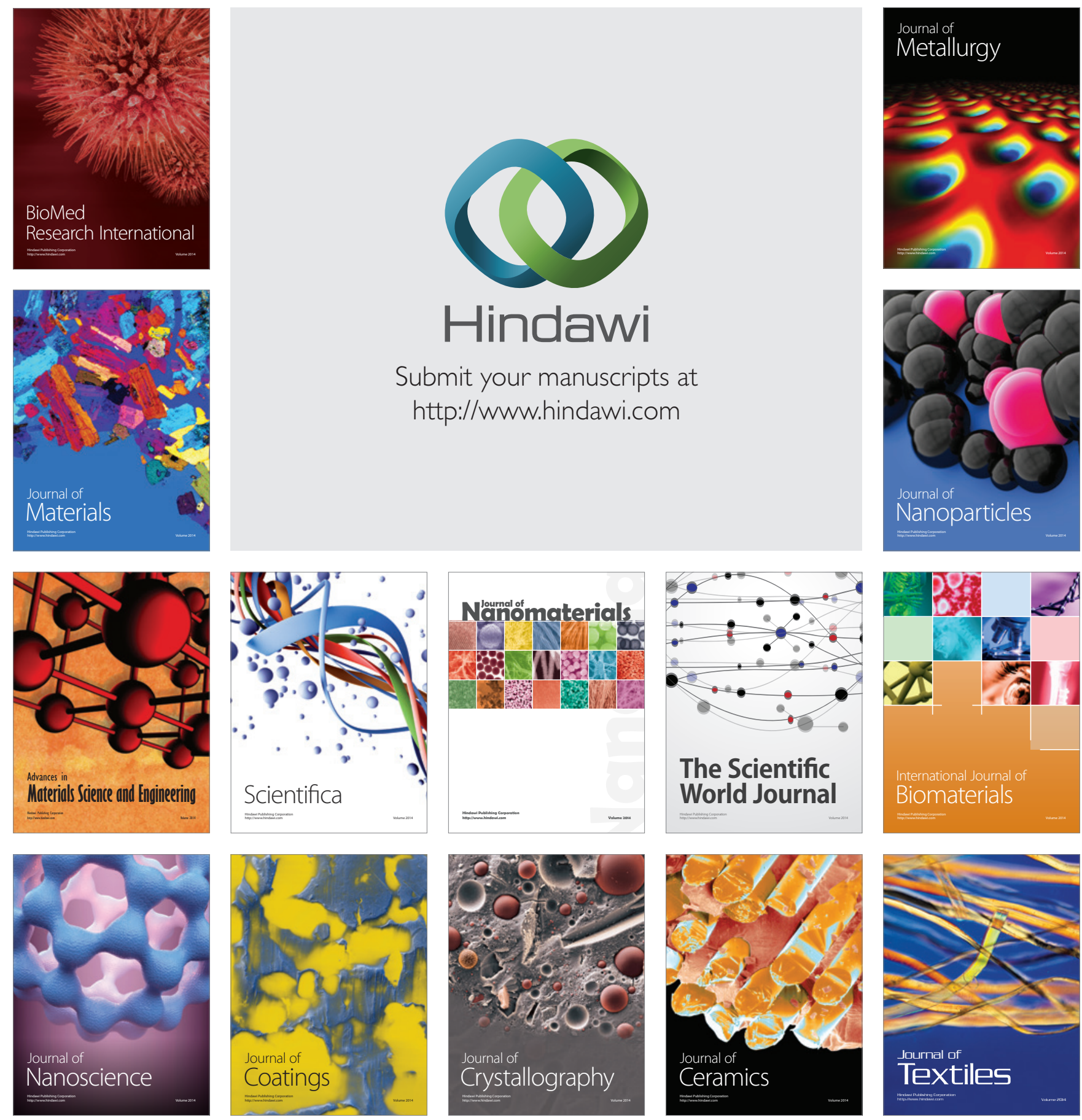